\title{
THE MINIMUM SET, INFORMACIÓN, MATERIA Y SIGNIFICADO
}

\author{
THE MINIMUM SET, INFORMATION, \\ MATTER AND MEANING
}

\author{
ANDRÉS BRICEÑO GUTIÉRREZ \\ Centro de Innovación y Diseño Avanzado CINNDA \\ FabLab Santiago
}

Si a la realidad primaria y perceptible de la materia se le da forma sustancial a través de la información a la que se ve sometida, ¿en qué dimensión la materia y la tectónica de la obra arquitectónica se someten a ser informadas a través de herramientas digitales? El dilema es comprender el tiempo que nos rodea, la constatación de que estamos en medio de un proceso de cambio no consolidado del modelo, donde la tecnología y el conocimiento que la genera está más cercanos a lo cotidiano conquistando un lugar habitual en nuestra sociedad, ergo, modificando variables del proyecto arquitectónico.

materia, fabricación digital, información, bit-byte, origen, forma
Recibido: 4 de junio del 2019

Aprobado: 6 de agosto del 2019

doi: 10.26439/limaq2020.n006.4823

If the primary and perceptible reality of matter is substantially formed by the information to which it is subjected, in which dimension are matter and tectonics of the architectural work subjected to be informed by digital tools? The dilemma is to understand the time around us, the realization that we are in the middle of a process of unconsolidated change of the model, where technology and knowledge that generate it are closer to everyday life, conquering a usual place in our society, ergo, modifying variables of the architectural project.

matter, digital fabrication, information,

bit-byte, origin, form 
I TRÁNSITO MODERNO

Lo que hoy está probado, en otro tiempo era solo imaginado.

William Blake

A finales del siglo xviII, en los albores de la Primera Revolución Industrial y con más intensidad ya adentrados en el siglo xIx, en la ciudad comenzaron a aparecer los primeros indicios de un crecimiento urbano detonado por las migraciones que el nuevo mundo prometía. Particularmente, la condición urbana se definía por estructuras sociales verticales orientadas a la productividad y por el fortalecimiento de una naciente doctrina económica ortodoxa. En su conjunto, diseminaron lógicas de crecimiento en función de barrios dependientes de una industria en particular, emergiendo la idea del "barrio obrero" y, consecuentemente, el imaginario del "suburbio".

El oficio escaló a industria o más bien, ésta última lo absorbió, demandando, por consecuencia, mano de obra que asumiese los requerimientos productivos del nuevo mundo, creando necesidades que la ciudad y la arquitectura propiamente no habían recibido. El desafío, paulatinamente, demandó dosis de eficiencia y cobertura, parámetros que no necesariamente eran parte de la ecuación convencional en la creación arquitectónica que estaba profusamente centrada en debates estilísticos aún dependientes de la beaux arts.

Gradualmente, el desafío fue aumentando en complejidad, abriendo preguntas que no necesariamente estaban vinculadas con los valores estéticos y estilísticos acostumbrados a relacionarse con necesidades puntuales e individuales, trasladando las preguntas a un escenario en el cual las definiciones técnicas para conseguir eficiencia en los recursos, tiempos y criterios espaciales fueron ampliando la mirada de la arquitectura, fundándose paulatinamente en los alcances contemporáneos de la especialidad. Esa demanda por escalamiento que el escenario ejerció escalonadamente hizo transformar intensamente el espacio reflexivo de los arquitectos, transfiriéndoles la oportunidad para pensar en sistemas de mayor complejidad social y política.

Los hechos posteriores demandaron aún mayores niveles de complejidad al iniciar el siglo $\mathrm{xx}$, momento en el que comenzaba la curva de crecimiento exponencial en todos los niveles. De este modo, se creó un escenario en que los grandes conflictos sociales se abrieron paso permitiendo que se amalgamase una escena de arquitectos, diseñadores, artistas, científicos e intelectuales sobre la que se fue construyendo el pensamiento "moderno". El conflicto social es un hecho fundamental de la causa intelectual que creó parámetros que definieron las preguntas que delinearon lo contemporáneo, sintetizándose en constructos teóricos, pero con particular y prolífica práctica. El fenómeno social, encumbrado por la defensa de nuevos derechos civiles, dio paso a una arquitectura que asimiló la coyuntura a través del equilibrio de la dualidad técnica y creativa, liberándola de cuestiones que reconocían el clima sociocultural, abriendo paso a conceptos que sofisticaron la abstracción del hecho 
arquitectónico situándolo en un nuevo nivel de complejidad y vinculándole con preguntas orientadas a lo sistémico y no solo a lo objetual; "La verdadera forma presupone una vida verdadera. Pero ninguna vida pasada, ni tampoco ninguna vida imaginada. Este es el criterio. Por lo tanto, la pregunta ¿clasicismo o gótico? Es tan poco seria como sería la pregunta ¿constructivismo o funcionalismo?" (Mies Van der Rohe, 1927, p. 5).

Las demandas sociales de la época agruparon a masas de trabajadores en gremios que lucharon por sus derechos creando un lenguaje de conceptos que la nueva arquitectura paulatinamente fue considerando no solo como válidos, sino que hizo comprender que el rol final de la arquitectura era asumir esos desafíos como propios definiendo la gravedad que transformó completamente su sentido alejándola preliminarmente de la promesa del arte, situándola en un problema de espacio que debía responder a la nueva tecnología y sociedad. El desafío moderno se situó en las variables sistémicas que el pensamiento arquitectónico podía asumir, generando un espacio de reflexión cuestionando todos los principios referidos al oficio, enmarcando el proceso en parámetros fundacionales para operar en la conformación del espacio existencial moderno, sistematizado y reconociendo el proceso constructivo como una variable arquitectónica.

La modulación podría establecerse como el punto de partida del diseño arquitectónico sistematizado. Iniciado a principios de los años 30 , el advenimiento de la construcción modular sentó las bases de la concepción de un lenguaje y una gramática arquitectónica, contribuyendo a simplificar y racionalizar el diseño de edificios. (Chaillou, 2017)

Este devenir moderno expandió las posibilidades y creó parámetros sobre los cuales el ser humano se sitúa en el espacio y el entorno a través de códigos que adaptan su condición al nuevo escenario. En palabras de Norberg-Schulz (1980):

Básicamente se orienta (el ser humano) a objetos, es decir, se adapta fisiológicamente y tecnológicamente a las cosas físicas, influye en otras personas y es influido por ellas y capta las realidades abstractas o "significados" transmitidos por los diversos lenguajes creados con el fin de comunicarse. (Norberg-Schulz, 1980, p. 9)

En otras palabras, se opera a partir de la experiencia en el espacio que nos sostiene, se construyen jerarquías y estructuras a partir de la percepción de esa experiencia y, finalmente, se "da significado a su ambiente asimilándolo a sus propósitos al mismo tiempo que se acomoda en las condiciones que ofrece (el espacio)" (Norberg-Schulz, 1980, p. 12).

Pese a las indudables transformaciones estructurales que el modernismo generó en el tránsito entre los siglos XIx y xx, se infiere que la concretización del espacio existencial a través de la lectura del espacio arquitectónico moderno mantuvo el espesor del hecho arquitectónico dada la íntima correlación con que las circunstancias sistémicas fueron leídas por él. 
El interés por la ciudad era relevante, el modelo de acción que las élites poseían tenía interés pleno en el lugar que habitaban, en los territorios con los que se vinculaban productivamente; por lo tanto, existía una disposición a relacionarse con él y, a través de esto, valorar la ciudad desde lo público, desde lo identitario y local. El posterior desenlace, particularmente el de la posguerra, desencadenó una irrupción tecnológica que paulatinamente fue modificando la relación de las élites con sus territorios, con $s u$ ciudad o más bien su contexto, generando hechos que Bauman (2007) describió nítidamente:

\begin{abstract}
La separación de la nueva élite (asentada localmente pero con una orientación global y vinculada de una manera débil a su lugar de residencia) de los compromisos del pasado con la clase baja local, y la consiguiente brecha espiritual/comunicativa entre los espacios vitales/vividos de quienes se han separado y quienes se han quedado atrás, representan, sin duda, la novedad, más importante de carácter social, cultural y político asociada al paso del estado 'solido' de la modernidad al 'líquido' [...] Por este motivo, las ciudades contemporáneas son el escenario o el campo de batalla donde los poderes globales y los sentidos e identidades, obstinadamente locales, se encuentran, chocan, luchan y buscan un acuerdo satisfactorio, o al menos soportable, una modalidad de convivencia que pueda ser una paz duradera, pero que por lo general solo resulta un armisticio, breves intervalos para reparar las defensas dañadas y volver a desplegar las unidades de combate. (p. 113)
\end{abstract}

Las circunstancias, inevitablemente invitan a construir preguntas que necesariamente tienen necesidades que no soportan respuestas desde objetos sin calibre sistémico o al menos, sin comprensión de esta dimensión.

\title{
LATENCIA TECNOLÓGICA
}

El conocimiento - saber - y la comprensión - experiencia - se han visto favorecidos por la irrupción de la tecnología contemporánea creando un ecosistema que paulatinamente ha ampliado las opciones para que el anónimo pierda esa condición y la masa se transforme en un colectivo que potencialmente recupere su condición de comunidad. Michel Serres, reconoce una influencia crucial de la tecnología en la evolución de las ciencias exactas, pero no así en las ciencias sociales. La arquitectura, ¿en qué posición se siente más cómoda?, ¿como parte del cuerpo de conocimientos de las ciencias exactas vinculadas al construir o sociales vinculadas al significado? Podríamos inferir que su dimensión conceptual la hacen navegar por círculos hermenéuticos asociados a la búsqueda de respuestas para el ser humano, y, por otra parte, su dimensión técnica la lleva hacía cuestiones eminentemente definidas por las ciencias exactas. Este escenario dual es carne para la ceguera o el atrevimiento.

Es evidente que muchos cambios tecnológicos y sociales se han consolidado en torno a una secuencia de relaciones geométricas que enuncian potenciales alternativas de organización social, enunciando transformaciones radicales en los formatos sobre los cuales la arquitectura, el diseño y la ciudad hacen 
tangibles. En cierto modo, el medioambiente construido es un hecho que constata un modelo de organización social.

Internet ha generado transformaciones sustanciales en muy poco tiempo, manifestándose como un instrumento solo comparable con otras invenciones modernas, tales como la imprenta, la electricidad o la combustión. En la actualidad, pese a las transformaciones relevantes que este ha empujado en áreas tan trascendentes como la economía, el aprendizaje, la movilidad urbana, la producción entre otras, han emergido nuevas élites que han monetizado paulatinamente los espacios de libertad inicial, estableciendo códigos que limitan otras potenciales cambios estructurales. Leer los efectos de todas estas coyunturas, definir las variables esenciales sobre las cuales pensar el futuro de nuestra civilización atendiendo la inefable crisis social, cultural y ecológica que nos convoca, son parte de los desafíos generacionales.

Según Rifkin (2010), el hecho urbano y arquitectónico desde siempre ha estado vinculado directamente a los avances tecnológicos de cada época y los primeros asentamientos urbanos que concretizaron el sedentarismo se establecieron a partir de lo que las civilizaciones hidráulicas del rio Nilo (Sumatra) lograron alcanzar a través de la tecnología, conocimiento y logística adecuada para utilizar el agua como fuente de regadío. Estas culturas originaron los primeros sistemas organizados que convirtieron a las nuevas ciudades en imanes donde convergían y se mezclaban las culturas. Así se dio el inicio de una actitud cosmopolita. El proceso histórico nos ha llevado a través de un periplo de avances tecnológicos que han establecido un sistema social altamente complejo y que a veces por su rapidez impide asimilar su significado real. El acceso a una amplia gama de tecnologías el día de hoy permite visualizar operaciones que potencialmente originan transformaciones en la cultura, la sociedad y por supuesto en nuestro entorno construido. Es decir, los "parámetros de diseño" tienen directa correlación con las tecnologías que definen una época.

Suponer que la arquitectura está ausente de toda transformación económica, social y productiva sería desconocer el velo fino que separa al objeto arquitectónico del sistema y los consecuentes recursos con los que cuenta para su labor creativa, reflexiva y técnica.

En esencia, el lenguaje formal de la creación se hace carne, ya sea colocando una piedra sobre la otra o utilizando un software que radicaliza la precisión para vincularse con la realidad; el tema de fondo es qué tipo de preguntas o variables se introducen en la formulación del fenómeno proyectual.

El lenguaje arquitectónico muta a través de la incorporación de nuevos argumentos para definir la técnica constructiva, para modificar la corporalidad del espacio a través de nuevos materiales e incluso incorporar dinámicas sociales que definen su sentido. Es ahí, en ese cruce temporal, donde el arquitecto sitúa su esencia al problema de habitar. 
Así como la piedra en su minuto fue la materia sobre la cual su propia sumatoria derivó en la definición espacial de ese habitar, estableciendo una comprensión de las leyes físicas que le permitieron construir un lugar habitado, el hormigón armado fue el salto tecnológico que le posibilitó a la materia situarse en un estado maleable e igualmente permanente.

La materia (su estado y sus posibilidades), construye una realidad primaria íntimamente ligada con la información que regula la sustancia con la cual se le da forma, toma presencia y se somete.

El arquitecto de 'hoy', tiene el dilema de comprender el tiempo que nos rodea. Dicho de otro modo; hoy existe la constatación de que estamos en medio de un proceso de cambio no consolidado del modelo económico y productivo imperante, y es un hecho que la tecnología y el conocimiento que este genera, está más cerca de lo cotidiano conquistando un lugar habitual en nuestra sociedad. Hoy, paulatinamente las herramientas disponibles, sumadas a la distribución de la información, son capaces de trasladar la "sensación" de lo virtual al mundo físico, tangible. En el fondo, el mundo binario y relativo a números aparentemente sin sentido se transforma en una capacidad para dar forma sustancial a algo, en este caso a la materia.

Esta transmutación entre el dato y materia, genera una tensión que permite entender que la arquitectura puede ver modificada su tectónica, su estado a través de una materia previamente "informada". Una materia informada y numéricamente controlada puede ser una tentación para romper la convención de la arquitectura y situarse en una pornografía formal, o, en su defecto, ofrecer una oportunidad para abrir un flanco que permita vincular al pensamiento y producción arquitectónica con la libertad de una materia emergente que contribuya a comprender el tiempo que vivimos evidenciando un pensamiento sistémico por sobre el centrado en el objeto.

Digital materiality, como lo expresó Morizio (2014), ha emergido como "un concepto relativamente nuevo en la literatura de sistemas de información (SI) que intenta dar "sustancia" a, o explicar las propiedades del material de artefactos digitales. Estos artefactos, tales como softwares, están desafiando nuestras tradicionales suposiciones de lo que es la "materialidad", y que en términos arquitectónicos ha surgido como una abstracción que permite dar cuenta de las variables que se expanden en términos de diseño, particularmente a través de procesos que consideran la totalidad del hecho arquitectónico. Acuñado en esos términos (arquitectónicos) por Gramazio y Kholer (2008), digital materiality reconoce un estado incipiente en la arquitectura, definiéndole como:

Un término que designa una transformación emergente en la expresión de la arquitectura. Reconocemos que lo material se ve continuamente enriquecido con características digitales, y estas características afectan significativamente la naturaleza material de la construcción de arquitectura. Digital materiality emerge a través de la interacción entre el proceso digital y material durante el diseño y construcción. La síntesis de estos dos aparentemente distintos mundos — digital y material - da lugar a 
evidentes nuevas realidades y atractivos. Materiales y datos, programación y construcción se entretejen unos a otros. La fabricación digital posibilita esta síntesis. Le permite al arquitecto de hoy en día, entrelazar un diseño de datos directamente en el proceso constructivo material. De esta forma, el material es enriquecido con información, es "informado". En el futuro, los arquitectos podrán intervenir con profundidad en procesos de fabricación digital y participar formándolos inclusive. Este es un escenario nuevo: se transforman las posibilidades, y con ello, el marco profesional del arquitecto. (Gramazio y Kohler, 2008, p. 7)



Figura 1. SandSTONED 2

\section{Elaboración:} Gulfer, Atripaldi, Briceño-Gutiérrez, Khosrovani

Evidentemente, tales circunstancias no hacen más que incorporar nuevas herramientas para intervenir el proceso sobre el cual se establecen los parámetros formales ampliándolos, particularmente, a causa de una relación más íntima con el procedimiento de manipulación formal con la materia; es decir, desde la comprensión del sistema que genera el objeto y no desde el objeto como conclusión formal. El riesgo de tales circunstancias es aplicar el proceso desde la agitación que provoca el estado latente y potencial de poder conquistar cualquier forma posible evitando la pregunta inicial, alejándose del hecho físico, una decisión constructiva que convoca la acción de habitarle. Peligroso es a su vez, porque los procesos de diseño son tentados por la forma como objetivo primordial e inclusive justificando todo a través de la deformación de conceptos que distorsionan el significado del espacio arquitectónico y al modelo que sustenta el qué y cómo habitamos en sociedad. 
Reflexionar sobre la oportunidad emergente que estas circunstancias otorgan a la definición física y espacial del cuerpo arquitectónico, particularmente a sus procesos, puede situarlo en un estado que permita a la obra, superar la fragilidad del éxtasis tecnológico reconociendo el potencial vinculante con su entorno social capturando su energía como una fuente vital no solo para incluirla en parámetros de diseño iniciales, sino para verificar su proceso de término de la obra. Ortega y Gasset (1983), dice que:

\begin{abstract}
El hecho de que el hombre habite donde quiera, su planetaria ubicuidad, significa, claro está, que carece propiamente de hábitat, de un espacio donde, sin más pueda habitar. Y en efecto, la tierra es para el hombre inhabitable. Para poder subsistir intercala entre todo lugar terrestre y su persona, creaciones, técnicas, construcciones que deforman, reforman y conforman la tierra, de suerte que resulte más o menos habitable [...] El hombre es un intruso en la llamada naturaleza. Viene de afuera de ella, incompatible con ella, esencialmente inadaptado, por eso construye. (p. 617)
\end{abstract}

El camino que ofrece la democratización tecnológica asociada a nuevos formatos de fabricación que exploran vehículos de control, adaptación, manipulación y vínculo con la materia abren velos para estados emergentes de ella, estableciendo marcos de acción eventualmente especulativos, pero que, particularmente, exponen un potencial para que "lo informado" también se alinee con las circunstancias de nuestra época: nuevos sistemas, no nuevos objetos aislados.

\title{
THE MINIMUM SET
}

La celeridad propuesta por la irrupción tecnológica en todos los ámbitos de nuestra vida ha sido tal que el espectro organizacional no logra comprender el alcance integral de los fenómenos que acontecen. En otras palabras, vivimos en un proceso de transformación sociocultural casi continuo - sin sentido de pausa - criando un escenario de alta incertidumbre en donde lo más evidente se hace indescifrable al momento de proyectar cualquier escenario futuro. $\mathrm{La}$ ambigüedad de las transformaciones radica, fundamentalmente, en la alta volatilidad de todas las relaciones que estructuran nuestra sociedad moderna, esto es, una realidad definida por variables que abren perspectivas que se presentan como beneficiosas, pero que no hacen eco en donde se radican. La progresión de la complejidad sistémica ha sostenido crecimientos exponenciales - Ley de Moore inclusive- amplificando a tales niveles el enjambre social que hoy el sentido de tiempo, lugar y por consecuencia espacio han sido trastocados. Podríamos aventurarnos a que todo tienda a cero.

El debate en torno a las consecuencias del avance tecnológico ha puesto sobre el tapete las circunstancias sobre las cuales la automatización puede hacerse cargo de una labor históricamente asociada al ser humano, evidenciando que las labores operativas, lineales, predecibles y alejadas de procesos creativos son susceptibles de ser reemplazadas por un proceso automatizado. Por contrapartida, en tareas erráticas, hermenéuticas marcadas por herramientas definidas por la creación, existe menor posibilidad de que se desplace la presencia 
humana; en conclusión, a mayores herramientas creativas, menos posibilidades, a menores de ellas, más posibilidad (Bakhshi, Frey y Osborne, 2015). El conflicto sistémico, subyace en la alta implicancia que el modelo productivo neoliberal ha generado en las herramientas hermenéuticas propias de los procesos creativos, centrando sus demandas en tareas operativas, lineales y que no aceptan al error como un antecedente válido, creando secuencias programadas donde se conoce el inicio y el término de los procesos, construyendo de esta forma tareas productivas en prácticamente todas las áreas del quehacer humano.

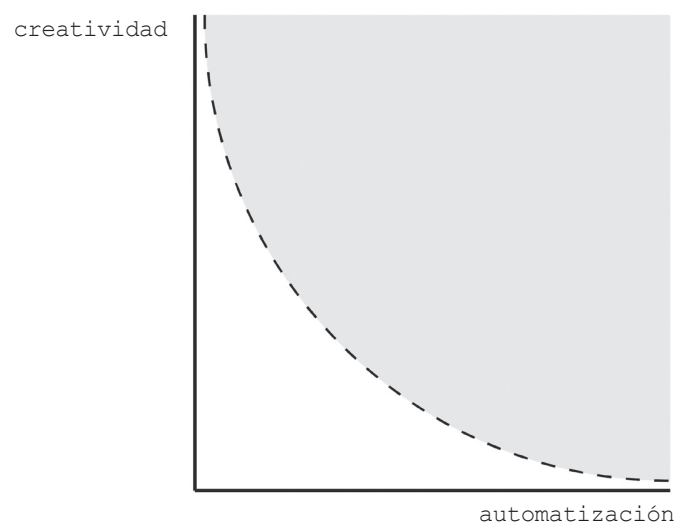

Figura 2.

Gráfico crea-

tividad versus

automatización

\section{Elaboración}

propia

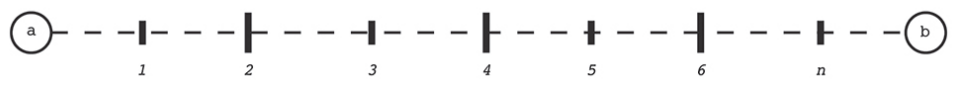

Figura 3.

Gráfico pensa-

miento lineal

LÍNEA CONTINUA

Se conoce el inicio y el término,

Elaboración

se establece como un proceso operativo que busca eficiencia y no acepta errores.

propia



Figura 4.

Gráfico

pensamiento

hermenéutico

\section{Elaboración}

propia

LÍNEA DISCONTINUA

Se conoce la pregunta inicial y no el resultado final, se define como un proceso hermenéutico que acepta y valoriza el error. 
La arquitectura y todo desarrollo centrado en un pensamiento desde el diseño, es decir proyectual, se ha forjado en la búsqueda de un equilibrio capaz de combinar una realidad técnica, funcional, pragmática o racional con una marcada inclinación por el significado, el arte o lo espiritual.

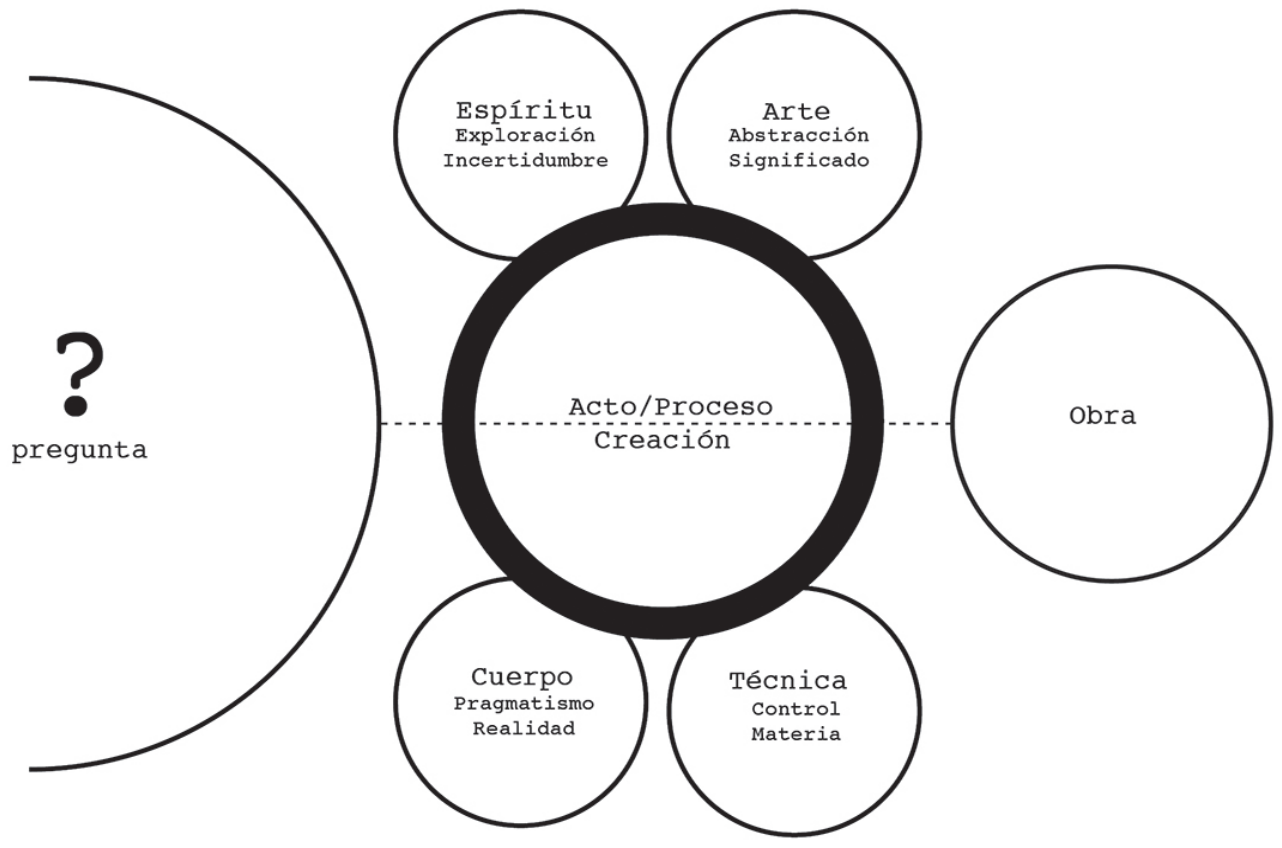

Figura 5. Este equilibrio es capaz de situar el lugar arquitectónico en un mensaje que consGráfico equilibrio truya experiencias significativas, circunscriba una cultura particular y que habite obra un territorio a través de hechos que se traducen en virtudes. Lamentablemente, la incesante demanda del mercado inmobiliario ha generado que una gran propor- 
arquitectónicos utilizando una base de datos (información), que categoriza y cataloga posibilidades aun rudimentariamente, pero con alcances concretos en un fin inevitable: desligarse del arquitecto. Aplicaciones y softwares como My Plan, Homestyler, Floor Plan o plataformas más sofisticadas como BIMx de Graphisoft, todo lo que los laboratorios de Autodesk ${ }^{1}$ están probando y notables avances a través de AI introduciendo data science en los procesos constructivos y eventualmente arquitectónicos, cuestionan al arquitecto desde la perspectiva productiva, es decir, en lo que los procesos lineales dan cuenta de su propia producción, pues no así en la arquitectura como tal.

En los años que se gestó el modernismo, uno de los factores que tomó realce en el desenlace arquitectónico, fue poner en valor el sentido de la construcción como tal, y desde ahí, leer la función como un ejercicio que albergaría la virtud del ser humano. La modulación de esta realidad dio paso a secuencias que asumían una realidad más pragmática que quería absorber las circunstancias de la época desde un hecho arquitectónico situado en lo político y social.

Este pragmatismo, en ningún caso, restó valor al significado del hecho arquitectónico, creando obras que han sido capaces de definir el mundo moderno, consolidándose como hechos atemporales.

Pensar en la posibilidad de reemplazar al arquitecto de hoy por un software no hace más que definir un proceso que justamente nace en hechos pragmáticos que inicialmente modularon la operación arquitectónica, pero que hoy han dado pie a la personalización, superficies complejas. E inclusive la independencia de la operación arquitectónica desde la emergencia propuesta por la inteligencia artificial, son fruto de una serie de hechos desde la "modulación, diseño computacional, parametrización e inteligencia artificial: esta secuencia de cuatro períodos refleja la cronología del progreso que, paso a paso, ha ido configurando y refinando los medios y métodos arquitectónicos" (Chaillou, 2017).

Presagiar el futuro del proceso de diseño a través de estas líneas es poco procedente, pero al aventurarse ante una realidad incuestionable, las reflexiones recaen en la cualidad y/o virtud del ser humano en relación con las nuevas herramientas; es decir, el argumento que permite vislumbrar un espacio de acción trascendental en el espesor del quehacer arquitectónico se posa sobre el "error" y el "significado" del lenguaje. La inteligencia artificial no es capaz de generar significado, no es capaz de reemplazar la incertidumbre del error en clave de virtud u elogio. Aquí, en este espacio prodigioso de síntesis, yace la singularidad del desafío: disponer de una formulación que permita la definición de procesos de diseño desde un punto de partida irreductible y con pensamiento sistémico. Un set mínimo de parámetros que construyan una estructura con significado que posteriormente se vinculará con una secuencia de acciones que permitirá que otro termine el proceso. Un set mínimo de parámetros que permita perder el control del proceso, a sabiendas que ese set inicial reconoce el error, el significado y la necesidad de la época.

1 Autodesk Labs, Autodesk Research 
Vivimos tiempos turbulentos, tiempos que demandan gestas contraculturales, que enfrenten la desintegración social y eviten concentrar el foco de los procesos en objetos aislados, cuerpos cerrados, transaccionales que no necesariamente reconocen tales demandas. Pensar en un hacer holístico viable, orientado al nuevo mundo que necesariamente debemos diseñar es el único rincón sobre el cual será posible disponer de los ingredientes sobre los cuales cualquier motor posterior procesará un ejercicio dónde emanará el hecho arquitectónico. En otras palabras, el espacio fenomenológico del arquitecto del mañana consistirá en disponer de un set de variables que alimenten una estructura con significado que sea capaz de crear sistemas, de comprender que el rol debe perder el control total del proceso, entender el sentido político de la obra a partir de una secuencia de acciones que viabilicen el sistema en su conjunto y no orientar todo el esfuerzo a objetos cerrados que desconocen la crisis. Hoy debemos diseñar para la crisis.

\section{REFERENCIAS}

Bakhshi, H., Frey, C. B. y Osborne, M. (abril de 2015). Creativity vs. Robots The Creative Economy and The Future of Employment. Nesta, 4(38). Recuperado de https://www.nesta.org.uk/report/creativity-vs-robots/

Bauman, Z. (2007). Tiempos líquidos, vivir en una época de incertidumbre. Buenos Aires: Tusquets Editores.

Blake, W. (2002). Prosa escogida. Barcelona: Dvd Ediciones.

Chaillou, S. (2017). The Advent of Architectural Al, a Historical Perspective. Toward Data Science. Recuperado de https://towardsdatascience.com/ the-advent-of-architectural-ai-706046960140

Gramazio, F.y Kohler, M. (2008). Digital Materiality in Architecture. Baden: Lars Müller.

Morizio, P. (2014). Conceptualising Digital Materiality and its Socio-Technical Implications through the Phenomenon of Crowdsourcing. Journal of Systems Integration, 4. Recuperado de http://www.si-journal.org/index.php/ JSI/article/viewFile/210/238

Neumeyer, F. (2000). Mies Van der Rohe. La palabra sin artificio. Reflexiones sobre la arquitectura 1922/1968. Madrid: El Croquis Editorial.

Norberg-Schulz, C. (1980). Nuevos caminos de la arquitectura, existencia, espacio y arquitectura. Barcelona: Editorial Blume.

Ortega y Gasset, J. (1983). Obras completas. Madrid: Alianza Editorial.

Rifkin J. (2010). La civilización empática. La carrera hacia una conciencia global en un mundo en crisis. Barcelona: Editorial Paidós.

Sassen S. (2017). The City: A Collective Good? The Brown Journal of World Affairs, 2, 119-126. Recuperado de http://saskiasassen.com//PDFs/ BrownJn|2017\%20City\%20Colectv\%20good\%20copy.pdf 